\title{
The Red Rectangle: Solid State Components of Varying Composition in the Outflow
}

\author{
F. Markwick-Kemper ${ }^{1}$, J. D. Green ${ }^{2}$, and E. Peeters ${ }^{3}$ \\ ${ }^{1}$ Department of Astronomy, University of Virginia, P.O. Box 3818, \\ Charlottesville, VA, 22903-0818, USA \\ email: ciska@virginia.edu \\ ${ }^{2}$ Department of Physics and Astronomy, University of Rochester, Rochester, NY 14627, USA \\ ${ }^{3}$ NASA Ames Research Center, MS 245-6, Moffett Field, CA 94035, USA
}

\begin{abstract}
We report the discovery of broad mid-infrared resonances in the outer regions of the Red Rectangle outflow (Markwick-Kemper et al. 2005). The peak position and the strength of the resonances vary spatially, but the full width at half maximum, as well as the shape of the feature, appears remarkably constant. While emission due to polycyclic aromatic hydrocarbons (PAHs) is also present at these locations, we show that PAHs cannot be the carriers of these new components. Instead, we argue that these resonances are caused by solid state components, perhaps simple Mg-Fe-oxides. The presence of such O-rich species in the otherwise C-rich outflows further complicates the picture of the formation and chemistry of the Red Rectangle nebula.
\end{abstract}

Keywords. infrared: stars - ISM: dust, extinction - ISM: individual (HD 44179; Red Rectangle) — stars: AGB and post-AGB — stars: circumstellar matter

The Red Rectangle is a relatively nearby system (estimates range from 300-700 pc), consisting of a post-AGB star HD 44179 with a circumstellar disk and a biconical outflow. The disk is seen almost exactly edge-on, providing superb conditions to study the emission from the outflows. The system shows a remarkable chemistry. Besides the so-called Extended Red Emission (Schmidt et al. 1980), for which the carrier is still unknown, there are emission features related to diffuse interstellar bands (Scarrott et al. 1992), and a wealth of PAHs detected in the outflow (Bregman et al. 1993). In contrast, the circumstellar disk contains predominantly oxygen-rich silicate dust (Waters et al. 1998). Optical imaging obtained with the Hubble Space telescope reveals that the biconical outflow shows a large amount of substructure, tracing variations in the physical conditions (Cohen et al. 2004). The question arises whether these variations in the physical conditions give rise to differences in the chemical composition of the outflows.

We have obtained infrared spectroscopy from $10-19.5 \mu \mathrm{m}$ at a resolution of $R \sim 600$ using Infrared Spectrograph (IRS) aboard Spitzer. We looked at three different pointings, about $30^{\prime \prime}$ away from the central star, in the northern outflow. The high-resolution slits are 5 pixels long, while the point spread function is $\sim 2-3$ pixels wide. We have performed sub-slit extractions with a width of three pixels. Figure 1 shows spectra obtained at pointings inside and outside the biconical shape of the outflow. Besides the PAHs emission around $11 \mu \mathrm{m}$, there are two very strong features present at $\lambda>13 \mu \mathrm{m}$ : one at $13-16 \mu \mathrm{m}$ and one longwards of $17 \mu \mathrm{m}$. The peak position and the relative strength vary spatially, while the FWHM and the shape remain remarkably constant.

We rule out that the feature is due to PAHs (Markwick-Kemper et al. 2005). Instead, we argue that it is due to a solid state carrier. A possible explanation of the $\lambda>17 \mu \mathrm{m}$ features could be provided by simple Mg-Fe-oxides. Figure 1 shows the comparison with laboratory spectroscopy (Henning et al. 1995). The change in peak position could be 

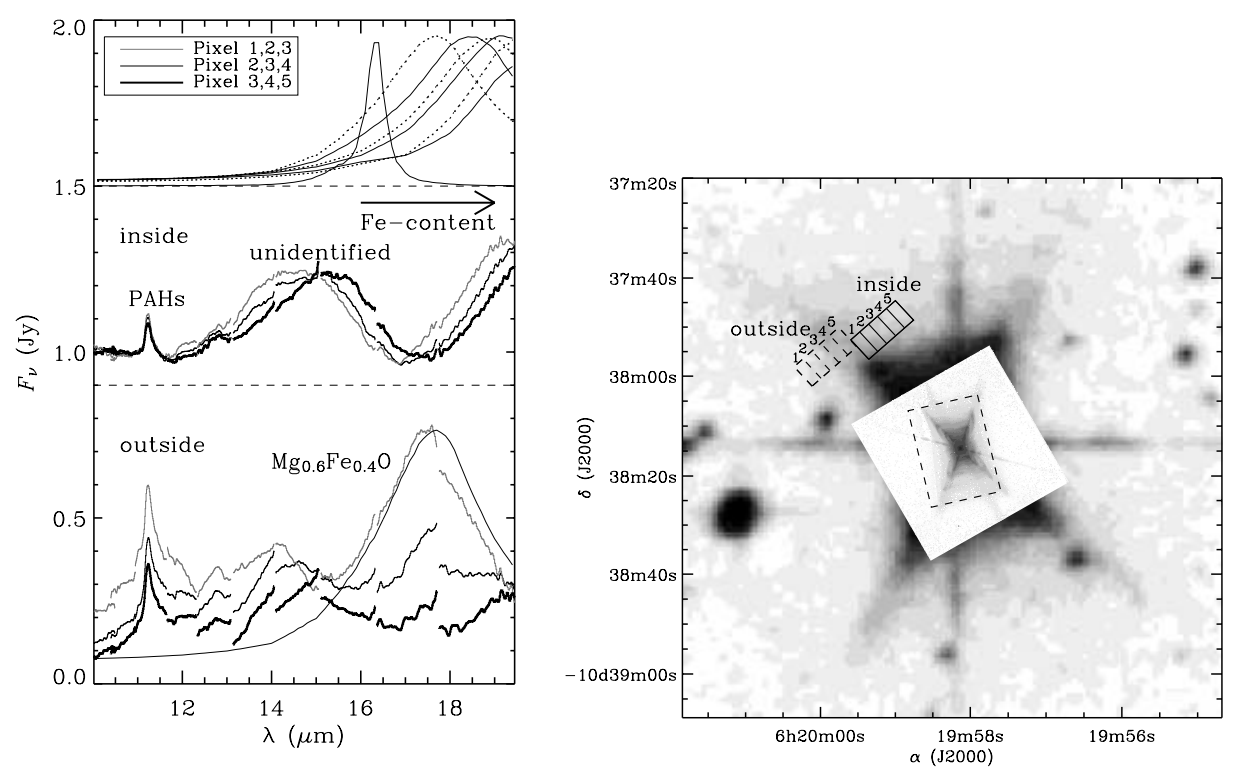

Figure 1. Left: IRS high resolution spectra (bottom two panels) compared with the laboratory spectroscopy of simple oxides of varying composition (top panel). The map on the right shows the slit and pixel positions of these observations.

explained with a change in composition. The features at 13-16 $\mu \mathrm{m}$ remain unidentified, but we are looking into mixtures of the simple oxides with either spinels or silicates.

The presence of oxides in the C-rich outflow of the Red Rectangle paints a complex picture of circumstellar chemistry, as does the suggested presence of PAHs in the disk (Vijh et al. 2005). The origin of the O-rich species in the outflow is unclear. It could be a relic from earlier mass loss, or it could be caused by stellar wind erosion of the circumstellar O-rich disk. Moreover, the varying composition is yet to be explained.

\section{References}

Bregman, J.D., Rank, D., Temi, P., Hudgins, D., \& Kay, L. 1993, Ap. J. 411, 794

Cohen, M., Van Winckel, H., Bond, H.E., \& Gull, T.R. 2004, A. J. 127, 2362

Henning, T., Begemann, B., Mutschke, H., \& Dorschner, J. 1995, A\& AS 112, 143

Markwick-Kemper, F., Green, J.D. \& Peeters, E. 2005, Ap. J. 628, L119

Scarrott, S.M., Watkin, S., Miles, J.R., \& Sarre, P.J. 1992, MNRAS 255, 11P

Schmidt, G.D., Cohen, M., \& Margon, B. 1980, Ap. J. 239, L133

Vijh, U.P., Witt, A.N., \& Gordon, K.D. 2005, Ap. J. 619, 368

Waters, L.B.F.M., Waelkens, C., van Winckel, H., et al. 1998, Nature 391, 868

\section{Discussion}

Bowey: Have you considered temperature shifts for the origin of the finer detail changes in the $14-16 \mu \mathrm{m}$ feature positions between slit pointings?

MARKWICK-Kemper: Yes, we have. Besides composition, such effects as temperature changes and grain properties affect the spectral appearance. However, temperature specific optical properties are not yet available for the oxides. 